\section{PWE-076 ABSENCE OF GUT MICROBIOTA REDUCES LIPOPOLYSACCHARIDE-INDUCED EPITHELIAL CELL SHEDDING IN THE SMALL INTESTINE}

${ }^{1} \mathrm{~K}$ Hughes ${ }^{*},{ }^{1} \mathrm{C}$ Alcon-Giner, ${ }^{2} \mathrm{M}$ Lawson, ${ }^{2} \mathrm{~K}$ McCoy, ${ }^{2} \mathrm{~A}$ Macpherson, ${ }^{1} \mathrm{~L}$ Hall, ${ }^{1} \mathrm{~A}$ Watson. ${ }^{1}$ Medicine, University of East Anglia, Norwich, UK; ${ }^{2}$ Maurice Müller Laboratories, University of Bern, Bern, Switzerland

\subsection{6/gutjpl-2014-307263.336}

Introduction Cell shedding, the process by which intestinal epithelial cells (IECs) are extruded from the small intestinal (SI) villus, is known to be elevated in patients with inflammatory bowel disease (IBD) and is correlated with disease relapse. Importantly, the epithelial barrier is in contact with intestinal bacterial communities (i.e. the microbiota) and studies have correlated disturbances in the microbiota with IBD. Thus, we hypothesised that cell shedding may be modulated by the microbiota.

Methods Specific pathogen free (SPF) and germ-free (GF) C57BL/6 mice, including GF mice reconstituted for 4 weeks with altered Schaedler flora (ASF), stable Defined Moderately Diverse Microbiota (sDMDMm2), or SPF faeces, were given $10 \mathrm{mg} / \mathrm{kg}$ Lipopolysaccharide (LPS) intraperitioneally to induce SI cell shedding. Animals were euthanized $1.5 \mathrm{~h}$ post-LPS. Tumour Necrosis Factor- $\alpha$ (TNF- $\alpha$ ) and cleaved caspase 3 (CC3) ELISA were performed on whole SI homogenates. Immunohistochemistry (IHC) for CC3 was performed on formalin fixed paraffin embedded SI tissue and CC3 +ve villus IECs quantified using WinCrypts and Score programs. Statistics were performed using ANOVA with Dunnett's post-test.

Results ELISA analysis showed significant decreases in CC3 in GF vs SPF mice following LPS administration (2.6-fold $+/$ $0.3, \mathrm{p}<0.01)$. Decreased levels of TNF- $\alpha$ (GF, 189+/$56 \mathrm{pg} / \mathrm{ml}$ vs SPF, $548+/-66$; p < 0.01), showed a potential mechanistic basis for this change. Reconstitution with ASF or sDMDMm2 failed to restore levels of shedding observed in LPS treated SPF mice. CC3 ELISA (ASF, 1.1-fold+/-0.3, ns; sDMDMm2, 1.0-fold+/-0.1, ns); TNF- $\alpha$ ELISA (ASF, 311+/$48 \mathrm{pg} / \mathrm{ml}, \mathrm{p}>0.05 ; \mathrm{sDMDMm} 2 ; 231+/-77 \mathrm{pg} / \mathrm{ml}, \mathrm{p}<$ $0.05)$. IHC and count analysis confirmed that LPS treated GF, ASF or sDMDMm2 mice were unable to mount a normal cell shedding response vs LPS treated SPF mice: Mean\% CC3 positive IECs along the length of the villus of $4.3 \%+/-$ $1.2,0.6 \%+/-0.3,0.5 \%+/-0.2$ vs $7.9 \%+/-1.1$, respectively (all $\mathrm{p}<0.01)$. Importantly, when LPS was delivered to GF mice reconstituted with SPF faeces, similar rates of shedding to LPS treated SPF mice were observed and TNF- $\alpha$ production was restored.

Conclusion GF mice are largely refractory to LPS induced cell shedding, when compared to SPF or fully reconstituted GF mice, via modulation of the pro-inflammatory cytokine TNF- $\alpha$. These data strongly implicate the intestinal microbiota in cell shedding and may help to shape microbiota-based treatment of IBD patients.

Disclosure of Interest None Declared.

\section{PWE-077 ACCESS TO A FAECAL CALPROTECTIN SERVICE PROVIDES CLINICIANS WITH THE CONFIDENCE TO DIAGNOSE AND TREAT CONCOMITANT FUNCTIONAL BOWEL SYMPTOMS IN KNOWN IBD PATIENTS}

K Bundhoo*, A Aravinthan, M Johnson. Gastroenterology, Luton and Dunstable Hospital, Luton, UK

10.1136/gutjnl-2014-307263.337
Introduction An accurate clinical assessment of disease activity in inflammatory bowel disease (IBD) is essential to provide appropriate management strategies. The concurrent presence of functional symptoms in IBD patients is common and said to occur in $80 \%$ of proctitis patients, $60 \%$ of UC patients and $40 \%$ of Crohn's patients. ${ }^{1}$ A high symptom index can strongly influence clinical assessment and expose patients to unnecessary investigations. Faecal calprotectin (FC) has a high negative predictive value of $96 \%$ for inflammation therefore allowing use in this cohort to differentiate functional and organic symptoms. ${ }^{2}$

Methods All FC data over a 2 year period was collected in IBD outpatients with a diagnostic uncertainty about symptoms being functional or organic in nature and whether further endoscopic examination was necessary. FC results were regarded as normal $(<50 \mu \mathrm{g} / \mathrm{g})$, borderline $(50-100 \mu \mathrm{g} / \mathrm{g})$ or positive $(>100 \mu \mathrm{g} / \mathrm{g})$ and correlated with endoscopic assessment and subsequent influence on management.

Results 262 FC measurements were performed in IBD patients where there was diagnostic uncertainty about symptoms being organic or functional in origin. In this cohort, unnecessary colonoscopy was spared in 83\% (218/262), including 62/66 with normal FC, 26/27 borderlines and 130/169 positives.

Despite a normal FC, 4 patients underwent further assessment via colonoscopy for routine surveillance with no evidence of active disease. In addition, some patients were investigated with CT colonography as an alternative assessment method. $0 / 2$ scans in the borderline group showed positive findings with 6 being performed in the positive FC group. Of these, 5 had active disease with 1 showing a psoas abscess requiring inpatient treatment.

As a result of a positive $\mathrm{FC}$, a direct change in management was made in 114/169 (67\%) without the need for further endoscopy. In the case of a negative FC result, 14/66 (21\%) patients had an alteration in their treatment regimes to focus upon targeting functional bowel symptoms.

Conclusion Faecal calprotectin measurement spared $80 \%$ of the colonoscopies being considered to assess symptomatic IBD patients. Both positive and negative results had a strong influence on subsequent management. FC measurement provides clinicians the confidence to isolate and manage functional symptoms in their IBD cohort, whilst preventing unnecessary treatment escalation. In those with a positive FC result, appropriate treatment could be initiated whilst avoiding the increased risks of endoscopy in acutely inflamed patients.

\section{REFERENCES}

1 Keohane, et al. Am J Gastroenterol. 2010 Aug;105(8):1788, 1789-94

2 Turvill, High negative predictive value of a normal faecal calprotectin in patients with symptomatic intestinal disease. Frontline Gastroenterology 2012;3:21-28

Disclosure of Interest None Declared.

\section{PWE-078 MEAN CORPUSCULAR VOLUME BUT NOT LYMPHOCYTE COUNT IS A PREDICTOR OF THIOPURINE DOSE ADEQUACY AND TOXICITY}

${ }^{1}$ A Kneebone, ${ }^{2} S S$ Poon, ${ }^{3} R$ Asher, ${ }^{3} R$ Jackson, ${ }^{1} B$ Gregg, ${ }^{2} S$ Kerr, ${ }^{1} \mathrm{P}$ Collins, ${ }^{1} \mathrm{C}$ Probert, ${ }^{1} S$ Subramanian, ${ }^{1} \mathrm{M}$ Dibb*. 'Department of Gastroenterology, Royal Liverpool and Broadgreen University Hospital, Liverpool, UK; ${ }^{2}$ Faculty of Medicine, University of Liverpool, Liverpool, UK; ${ }^{3}$ Medical Statistics, Liverpool CR-UK Centre, Liverpool, UK

\subsection{6/gutjnl-2014-307263.338}

Introduction The thiopurines, azathioprine (AZA) and mercaptopurine (MP), commonly used in the treatment of inflammatory 
bowel diseases (IBD), are typically dosed according to patient's body weight. A previous meta-analysis showed higher remission rates in patients with "therapeutic" levels of 6-thioguanine (6TGN), but weight based dosing correlates poorly with 6-TGN levels (1). 6-TGN testing is not universally available, results are not available immediately and repeated measurements are necessary to ensure dose adequacy and adherence to therapy. Proxy measures such as mean corpuscular volume (MCV) and lymphocyte count (LC) have been advocated as markers of dose adequacy. We aimed to analyse the relationship between 6-TGN levels, MCV, LC and other putative surrogate markers of therapeutic 6-TGN levels.

Methods This retrospective study was conducted at the Royal Liverpool University Hospital. All patients who had concurrent measurements of 6-TGN and full blood count were included in the analysis. 6-TGN levels were classed as sub-therapeutic $(<230)$, therapeutic $(230-450)$ or supra-therapeutic $(>450)$. The association between 6-TGN, patient demographics, MCV, LC and other putative surrogate markers was estimated using a multivariable linear regression model for continuous 6-TGN and a proportional odds logistic regression model for the ordered 6TGN levels. All results were declared statistically significant if $\mathrm{p}$ $<0.05$.

Results A total of 106 patients (48 male, 58 female) were included and contributed 133 measurements. Of these patients 58 (55\%) had Crohn's disease and 47 (44\%) had ulcerative colitis. The mean azathioprine dose was $123.5 \mathrm{mg}$ (SD 73.8) or $1.70 \mathrm{mg} / \mathrm{kg}$ (SD 0.67). After adjusting for other variables, a one unit increase in MCV, was associated with a 10.88 unit increase in 6TGN levels, Figure 1 (95\% CI: 7.63 and 14.014, p < 0.0001 ) and a one unit increase in ALT was associated with a 2.67 unit decrease in 6TGN levels (95\% CI: 0.36 to $4.97 \mathrm{p}=$ 0.0237). There was no correlation between LC, NC, WCC or ALKPHOS and 6-TGN levels.

Conclusion MCV and 6-TGN nucleotide levels increase together. If 6-TGN levels are not available, MCV can be used as a crude but imperfect surrogate marker of dose adequacy and toxicity.

\section{REFERENCE}

Osterman MT, Kundu R, Lichtenstein GR, Lewis JD. Association of 6-thioguanine nucleotide levels and inflammatory bowel disease activity: a meta-analysis. Gastroenterology 2006;130:1047-53

Disclosure of Interest None Declared.

\section{PWE-079 DEXA RATES AND OUTCOMES IN AN IBD POPULATION COHORT.. LESSONS LEARNT}

${ }^{1,2} \mathrm{MN}$ Shuhaibar ${ }^{*}{ }^{1,2} \mathrm{C} \mathrm{O}^{\prime}$ Morain. ${ }^{1}$ Department of Gastroenterology, Adelaide and Meath Hospital, Dublin, Ireland; 'Department of Clinical Medicine, Trinity College Dublin, Dublin, Ireland

\subsection{6/gutjnl-2014-307263.339}

Introduction Osteoporosis is one of the under diagnosed and undertreated conditions worldwide. It is estimated that 1 in 3 women and 1 in 5 men in Ireland are diagnosed with it above the age of 50 years and will be likely to become more common with the increased life expectancy.

In our study, we aimed to assess the rate of screening with DEXA scans for this condition in a prospective IBD cohort and to evaluate the results. We also studied possible factors that may influence DEXA rates.

Methods As part of two decades follow up study of a multicentre Irish IBD prospective cohort from the early 1990's that was part of European Collaborative Study on IBD (EC-IBD), patients were traced and interviewed. There were 126 IBD patients from the Greater Dublin area who consented and completed the study questionnaire, following Ethical committees' approvals. In this questionnaire we evaluated DEXA frequency and possible factors that may influence it; including patient diagnosis, age, gender, family history of IBD, courses of steroids, social class, total years of education, attending gastroenterologist or a surgeon, years of loss to follow up (LTFU) and membership to the national IBD patient support group (Irish Society of Crohn's Colitis ISCC).

Results were analysed with logistic regression and forward stepwise analysis using $\mathrm{R}$ program 3.0.1 software, taking $\mathrm{p}$ value $<0.05$ to be statistically significant.

Results Of the total 126 patients only 58 (46.03\%) patients had DEXA scans performed. Of those $67.44 \%$ had CD and $33.73 \%$ had UC. When evaluating DEXA results osteoporosis was diagnosed in 26.67 and $21.43 \%$ of CD and UC patients respectively. Osteopenia was diagnosed in $43.33 \% \mathrm{CD}$ and $42.86 \%$ of UC patients. About $30 \%$ of remaining scanned patients had normal DEXA. Logistic regression analysis showed that patients with positive family history of IBD and longer years of education were more likely to have had a scan with $\mathrm{P}=0.024$ and 0.049 respectively. Other possible risk factors didn't reach statistical significance. Patients who were less compliant and LTFU were less likely to get DEXA with $p=0.045$ in $C D$ and $p=0.0336$ in UC group.

Conclusion Osteoporosis is an increasingly recognised condition and recently has been considered as one of the extra intestinal manifestations of IBD. Patient and doctor awareness is paramount to screening and diagnosis. Our study showed that $\mathrm{CD}$ patients were more likely to have DEXA scans during their disease course. The rate of osteoporosis was higher in CD patients, despite their fewer numbers when compared to UC group. Over $40 \%$ of IBD patients had osteopenia. Early detection and appropriate management will help to reduce fracture risk, improve patient quality of life in a cost effective manner.

Disclosure of Interest None Declared.

\section{PWE-080 BEWARE THE INTERACTION BETWEEN THIOPURINES AND WARFARIN}

${ }^{1} \mathrm{M}$ Dadgar*, ${ }^{2} \mathrm{G}$ Pickford, ${ }^{2} \mathrm{P}$ MacCallum, 'D Rampton, 'L Langmead. 'Gastroenterology, The Royal London Hospital, London, UK; ${ }^{2}$ Haematology, The Royal London Hospital, London, UK

\subsection{6/qutinl-2014-307263.340}

Introduction It is not uncommon for patients to require both immunosuppression and anticoagulation and for warfarin and thiopurines to be prescribed concurrently. There is limited evidence that thiopurines can inhibit the actions of warfarin. This potential interaction needs to be addressed when monitoring patients established on warfarin as they commence thiopurines or change dose. IBD patients are increasingly changed to combination low dose thiopurine and allopurinol for high methylmercaptopurine: thioguanine nucleotide (MeMP:TGN) ratio to optimise thiopurine efficacy. However, allopurinol also may potentiate warfarin and this could further exacerbate the effect of Azathioprine/mercaptopurine dose lowering on warfarin activity.

Aim To raise awareness of risk of harm from a clinically important interaction between thiopurines and warfarin +/allopurinol. 